\title{
Approximation of Planar Rational Curves by Polynomial Curves
}

\author{
Zhang Hongmei \\ College of Computer Science and Technology \\ Inner Mongolia University For Nationalities \\ TongLiao, China \\ e-mail:Tongyu_007@163.com
}

\section{Zhang Zhigao}

College of Math

Inner Mongolia University For Nationalities

TongLiao, China

e-mail: zzgtongxin@163.com

\author{
Pei Zhili \\ College of Computer Science and Technology \\ Inner Mongolia University For Nationalities \\ TongLiao, China \\ e-mail:zhilipei@sina.com \\ *corresponding author
}

\begin{abstract}
A Rational curve $r(t)$ can pass through interpolation points $(m+1)$ by a polynomial of degree $m$ or under some circumstances, and the same is true when rational curve is restricted or the function values of $r(t)$ are replaced by derivatives. The paper will show in these cases a rational curve $r(t)$ of degree $m$ tends to pass through interpolation points more than $(m+1)$ geometric data. In a Hermite sense, this paper studied using polynomial interpolation of rational curve to reduce polynomial degree and to increase interpolation points by showing that a large class of rational parametric curves can be interpolated, in some certain cases, by a polynomial of degree m matching $2 m-2 k+4$ data. This paper constructs a simple polynomial $p$ (t) and employs quadratic planar rational curve as interpolation curve to test practicability of the method. The result shows that in test taking symmetrical points can effectively reduce computing time, improve approximation data and visual experience.
\end{abstract}

Keywords- Polynomial; Polynomial interpolation;Rational curve;Conic rational curve circle; Approximation

\section{INTRODUCTION}

The definition of polynomial interpolation shows that rational curve $\mathrm{r}(\mathrm{t})$ can be interpolated at $(\mathrm{m}+1)$ points by polynomial of degree $\mathrm{m}^{[1]}$. That is be proven that rational curve $r(t)$ tends to pass through interpolation points more than $(\mathrm{m}+1)$ when rational curve is restricted ${ }^{[2]}$. For instance, under certain circumstance when $\mathrm{m}$ is restricted as odd number, a segment of quadratic planar rational curve can be marked with points of $2 \mathrm{~m}$ by polynomial of degree $\mathrm{m}^{[3]}$. The paper further studies the above case of polynomial interpolation and experiments on quadratic planar rational curve by using Hermite interpolation to find quadratic planar rational curve with degree $\mathrm{m}$ and fitting points $2 \mathrm{~m}$ $2 \mathrm{k}+4$, of which, $\mathrm{k}$ is the sum of degree of planar rational curve.

Let $\mathbf{r}:[\mathbf{a}, \mathbf{b}] \rightarrow \mathbf{R}^{\mathbf{d}}, d \geq 2$, be the rational curve

$\mathbf{r}(t)=\mathbf{f}(t) / g(t)$
Where $\mathbf{f}$ and $g$ are polynomials of degrees at $\mathrm{M}$ and $\mathrm{N}$.

$$
k=M+N
$$

For each sample of parameter values : $a \leq t_{1}<t_{2}<\Lambda<t_{n} \leq b$, we construct a polynomial $p$ satisfying:

$p\left(t_{i}\right)=r\left(t_{i}\right) \quad \mathbf{p}^{\prime}\left(t_{i}\right)=u_{i} \mathbf{r}^{\prime}\left(t_{i}\right), \quad i=1,2,3 \ldots . . n(1)$

We make two assumptions to find the polynomials ${ }^{[4]}$ :

(A1) g has no zero point in $[a, b]$

(A2) g has no multiple roots (real root or imaginary root)

\section{CONSTRUCT INTERPOLATION POLYNOMIALS}

In order to satisfy interpolation polynomial $\mathrm{P}$ of (1.1), the approach is as follows:

Let $p(t)=r(t)+\lambda(t) w_{n}(t) r^{\prime}(t)$,

where $w_{n}(t)=\left(t-t_{1}\right)\left(t-t_{2}\right) \mathrm{K}\left(t-t_{n}\right)$

when $w_{n}\left(t_{i}\right)=0$, then $p\left(t_{i}\right)=r\left(t_{i}\right)$,to satisfy (A1),

when $\mu_{i}=1+\lambda(t) w_{n}^{\prime}\left(t_{i}\right)$, to satisfy(A2),

then solving polynomial equals to solving polynomial $\lambda(t)$.when $\lambda(t)$ is a specified polynomial; if a specified selection is made with in $\lambda(t)$, a polynomial $\mathrm{P}$ general type is obtained.

From $\left\{\begin{array}{l}p(t)=r(t)+\lambda(t) w_{n}(t) r^{\prime}(t) \\ \mathrm{r}(\mathrm{t})=\mathrm{f}(\mathrm{t}) / \mathrm{g}(\mathrm{t})\end{array}\right.$

to infer

$p(t)=\frac{g(t)-\lambda(t) w_{n}(t) g^{\prime}(t)}{g^{2}(t)} f(t)+\frac{\lambda(t) w_{n}(t)}{g(t)} f^{\prime}(t)$

Let $\left\{\begin{array}{l}\lambda(t)=g(t) x(t) \\ y(t)=\frac{1-x(t) w_{n}(t) g^{\prime}(t)}{g(t)}\end{array}\right.$

Put into (2.2)to get :

$w_{n}(t) g^{\prime}(t) x(t)+g(t) y(t)=1$

Then $p(t)=y(t) f(t)+x(t) w_{n}(t) f^{\prime}(t)$ 
let $p(t)$ satisfy(1.1), then $x(t)$ and $y(t)$ must satisfy(2.3). (2.3)can be simplified as:

$a_{0}(t) x(t)+a_{1}(t) y(t)=1$, which $a_{0}(t)=w_{n}(t) g^{\prime}(t)$, $a_{1}(t)=g(t)$

From algebra theory we know, if $a_{0}$ and $a_{1}$ are coprime, Euclidean algorithm can be used to solve polynomials $x$ and $y$, and to make sure the degrees of $x$ and $y$ are at most $d\left(a_{1}\right)-1$ and $d\left(a_{0}\right)-1$.So the preconditions of $\mathrm{A}(1)$ and $\mathrm{A}(2)$ must be considered to make sure $a_{0}$ and $a_{1}$ are coprime ${ }^{[5-6]}$.

Since $a_{0}(t)=w_{n}(t) g^{\prime}(t)=\left(t-t_{1}\right)\left(t-t_{2}\right) \mathrm{K}\left(t-t_{n}\right) g^{\prime}(t)$

$$
a_{1}=g(t)
$$

Then $d\left(a_{0}\right)=n+N-1, \quad d(y)=d\left(a_{0}\right)-1=n+N-2$

$$
d\left(a_{1}\right)=N, d(x)=d\left(a_{1}\right)-1=N-1
$$

Since $d(f)=M, d\left(f^{\prime}\right)=M-1$.

Then $d(p)=d(y)+d(f)=d(x)+d\left(w_{n}\right)+d\left(f^{\prime}\right)=n+N+M-2=n+k-2$

So polynomial $\mathrm{P}$ is set and it satisfies interpolation conditions $2(n+k-2)-2 k+4=2 n$.

Wherever Times is specified, Times Roman or Times New Roman may be used. If neither is available on your word processor, please use the font closest in appearance to Times. Avoid using bit-mapped fonts if possible. TrueType 1 or Open Type fonts are preferred. Please embed symbol fonts, as well, for math, etc.

\section{SOLVING POLYNOMIALS $x$ AND $y$ WITH EUCLIDEAN ALGORITHM}

In advanced algebra, Euclidean algorithm is used to get the greatest common factor for two arbitrary polynomials and it is also called division with remainder, which can be described as following ${ }^{[7]}$ :

Set $f(x), g(x)$ as two arbitrary polynomials, where $g(x) \neq 0$, use $g(x)$ to divide $f(x)$, to get $q_{1}(x)$, and remainder is $r_{1}(x)$;if $r_{1}(x) \neq 0$, use $r_{1}(x)$ to divide $g(x)$ and then to get $q_{2}(x)$, and the remainder is $r_{2}(x)$;if $r_{2}(x) \neq 0$, use $r_{2}(x)$ to divide $r_{1}(x)$, the result is $q_{3}(x)$, and the remainder is $r_{3}(x)$; continuous division gradually reduces the degree of result like the following: $d(g(x))>d\left(r_{1}(x)\right)>d\left(r_{2}(x)\right)>\Lambda$

Therefore after several divisions, there must be an occasion where the remainder is zero ${ }^{[8-9]}$, and then we get a series of equation:

$$
\begin{aligned}
& f(x)=q_{1}(x) g(x)+r_{1}(x), \\
& g(x)=q_{2}(x) r_{1}(x)+r_{2}(x), \\
& \ldots \ldots \ldots \ldots \ldots . \\
& r_{i-2}(x)=q_{i}(x) r_{i-1}(x)+r_{i}(x), \\
& \ldots \ldots \ldots \ldots \ldots \ldots \\
& r_{s-2}(x)=q_{s}(x) r_{s-1}(x)+r_{s}(x), \\
& r_{s-1}(x)=q_{s+1}(x) r_{s}(x)+0
\end{aligned}
$$

$r_{s}(x)$ and 0 share the greatest common factor $r_{s}(x)$. According to the previous description, $r_{s}(x)$ is the common factor for $r_{s}(x)$ and $r_{s-1}(x)$; Similarly, $r_{s}(x)$ is the greatest common factor for $f(x)$ and $g(x)$.

By processing the second equation from the bottom of the above equations, we get $r_{s}(x)=r_{s-2}(x)-q_{s}(x) r_{s-1}(x)$.

To put $r_{s-1}(x)=r_{s-3}(x)-q_{s-1}(x) r_{s-2}(x)$ into the above equation to eliminate $r_{s-1}(x)$, and to get $r_{s}(x)=\left(1+q_{s}(x) q_{s-1}(x)\right) r_{s-2}(x)-q_{s}(x) r_{s-3}(x) \quad$ By the same procedure, we eliminate $r_{s-2}(x), \Lambda, r_{1}(x)$ one after another, and then combine similar terms to get $r_{s}(x)=u(x) f(x)+v(x) g(x)$.

When $r_{s}(x)=1$, that is $u(x) f(x)+v(x) g(x)=1$, then the greatest common factor is 1 for $f(x)$ and $g(x)$.The following is the description on how to solve polynomials $X$ and $Y$ by using Euclidean algorithm.

Since $n \geq 1$ then $d\left(a_{0}\right) \geq d\left(a_{1}\right)$,for any $k$ in $k=0,1,2, \Lambda$,we can use $a_{k+1}$ to divide $a_{k}$ to find a remainder. Here Euclidean algorithm is illustrated by the following equations:

$a_{k}=q_{k} a_{k+1}+a_{k+2}$

Where $d\left(q_{k}\right)=d\left(a_{k}\right)-d\left(a_{k+1}\right)$, and $d\left(a_{k+2}\right)<d\left(a_{k+1}\right)$ ,when $a_{k+2}$ is a constant, the procedure is finished, set $r=k \quad, \quad a_{r}=q_{r} a_{r+1}+a_{r+2} \quad$,constant $\quad a_{r+2} \neq 0 \quad$ (if remainder $a_{r+2}=0$, then $a_{0}$ and $a_{1}$ share a common factor $a_{r+1}$, and they are not coprime; considering preconditions $\mathrm{A}(1)$ and $\mathrm{A}(2) \quad$ in(1.1),we know the constant is $\left.a_{r+2} \neq 0\right)$,we use(3.1)to reverse the procedure to get $\mathrm{X}$ and $\mathrm{Y}$ in (2.5).The reverse procedure is as following:

When $k=r$,

$$
a_{r+2}=b_{0} a_{r}+b_{1} a_{r+1}
$$

Where $b_{0}=1, b_{1}=-q_{r}$

When $k=r-1$, $a_{r+2}=b_{0} a_{r}+b_{1} a_{r+1}=b_{0} a_{r}+b_{1}\left(a_{r-1}-q_{r-1} a_{r}\right)=b_{1} a_{r-1}+b_{2} a_{r}$ Where $b_{2}=b_{0}-q_{r-1} b_{1}$

Process in this way,

When $k=0$,

$$
a_{r+2}=b_{r} a_{0}+b_{r+1} a_{1}
$$

Where $b_{j}=b_{j-2}-q_{r-j+1} b_{j-1}, \quad j=2,3, \Lambda, r+1$

Since $a_{r+2} \neq 0$, let $a_{r+2}$ divide(3.2)to get:

$1=\frac{b_{r}}{a_{r+2}} a_{0}+\frac{b_{r+1}}{a_{r+2}} a_{1}$

Combine (2.5) to get:

$$
X(t)=\frac{b_{r}(t)}{a_{r+2}}, \quad Y(t)=\frac{b_{r+1}(t)}{a_{r+2}}
$$

Here we analyze the degree of $x$ and $y$, from (3.3)we know:

$$
\begin{aligned}
d\left(b_{r}\right) & =d\left(q_{1}\right)+d\left(q_{2}\right)+\Lambda+d\left(q_{r}\right) \\
& =d\left(a_{1}\right)-d\left(a_{2}\right)+d\left(a_{2}\right)-d\left(a_{3}\right)+\Lambda+d\left(a_{r-1}\right)-d\left(a_{r}\right)+d\left(a_{r}\right)-d\left(a_{r+1}\right) \\
& =d\left(a_{1}\right)-d\left(a_{r+1}\right)<d\left(a_{1}\right)
\end{aligned}
$$

Combine (3.4)to get: $d(X)=d\left(b_{r}\right)<d\left(a_{1}\right)$ 
For the same reason $d\left(b_{r+1}\right)=d\left(a_{0}\right)-d\left(a_{r+1}\right)<d\left(a_{0}\right)$

Combine (3.4)to get: $d(Y)=d\left(b_{r+1}\right)<d\left(a_{0}\right)$

The uniqueness of $X$ and $Y$ can be analyzed as following : Suppose the existence of $\widetilde{X}, \widetilde{Y}$, which satisfies (6), then $\quad a_{0} X+a_{1} Y \equiv 1 \quad a_{0} \tilde{X}+a_{1} \tilde{Y} \equiv 1$

And then $a_{0}(X-\tilde{X})=a_{1}(\tilde{Y}-Y)$, Since $a_{0}$ and $a_{1}$ have no common factors, then $a_{0}$ cannot fully divide $a_{1}$, and then $a_{0} \mid(\tilde{Y}-Y)$, since $d(\tilde{Y}-Y)<d\left(a_{0}\right)$, then $\tilde{Y}-Y \equiv 0$.and then $\tilde{X}-X \equiv 0$

It can be inferred that(3.4)must be the only solution to the form(2.5)of $X$ and $Y$, and the greatest degrees are respectively $d\left(a_{1}\right)-1$ and $d\left(a_{0}\right)-1$ 。

The construction of interpolation polynomials $p$ is finished.

\section{APPROXIMATION OF CIRCLE}

The paper takes its experiment on approximation of circle to prove the theoretical hypothesis by showing the effect views and deviations.

\section{A. Parameter Equation of Circle}

As is knows, a regular circle with its center being the origin can be described as ${ }^{[10]}$ :

$$
\left\{\begin{array}{l}
x=\cos \theta=\frac{1-t^{2}}{1+t^{2}} \\
y=\sin \theta=\frac{2 t}{1+t^{2}}
\end{array}\right.
$$

Which $-\infty<t<+\infty$ Then, $r(t)=\frac{f(t)}{g(t)}=\frac{\left(1-t^{2}, 2 t\right)}{1+t^{2}}$

Analysis shows that except the point $(-1,0)$, all the other points of the curve are found on the circle with its center being the origin. To reduce the degree of numerator and denominator, a vector $(1,0)$ is added to $(4,1)$, then $r(t)=\frac{(2,2 t)}{1+t^{2}}$.

The center of the circle is $(-1,0)$ and all points are on the circle. Through transferring, it can be noticed that the degree of numerator for interpolation curve reduces by 1.the preconditions of(A1)and(A2) are satisfied because two roots of $\mathrm{g}(\mathrm{t})$ are $\sqrt{-1}$ and $-\sqrt{-1}$, and it has neither real root and nor multiple root in the region[a, b].

\section{B. Interpolation Polynomial for Circle}

From (2.4), interpolation polynomial P for circle can be written as: $p(t)=Y(t)(2,2 t)+X(t) \omega_{n}(t)(0,2)$

When solution to $\mathrm{X}$ and $\mathrm{Y}$ is known, the only polynomial $\mathrm{P}$ will be obtained .X and $\mathrm{Y}$ must satisfy: $a_{0} X(t)+a_{1} Y(t)=1$

which, $\quad a_{0}=\omega_{n} g^{\prime}=\left(t-t_{1}\right)\left(t-t_{2}\right) \Lambda\left(t-t_{n}\right) g^{\prime}$

$$
a_{1}=g
$$

Then $2 t \omega_{n}(t) X(t)+\left(1+t^{2}\right) Y(t)=1$

To infer: $d(X)=d\left(a_{1}\right)-1=2-1=1$,

$$
d(Y)=d\left(a_{0}\right)-1=n+1-1=n
$$

Then $d(p)=d(X)+d(\omega)=d(Y)+1=n+1$

Now , Euclidean algorithm can be applied to solving $X$ and $\mathrm{Y}$.By using $2 t \omega_{n}(t) /\left(1+t^{2}\right)$ to know that the completion of algorithm is determined by selecting interpolation point $t_{i}, \quad(1 \leq i \leq n)$.when a proper method is taken ,the procedure can be completed with efficiency . For instance, when interpolation points are taken symmetrically around 0 , the interpolation points are restricted in odd number ,the outcome is satisfying. Euclidean algorithm is efficient in simplifying procedure and reducing degree of $\mathrm{X}, \mathrm{Y}$ and $\mathrm{P}$ by $1^{[11]}$.The outcome meets the requirement of getting more interpolation points by less degree of polynomials. The outcome can be concluded as following:

Set $n=2 s=+1, \quad s \geq 0$,

Let $\left(t_{1}, \Lambda, t_{n}\right)=\left(-u_{s}, \Lambda,-u_{1}, 0, u_{1}, \Lambda u_{s}\right)$,

Which $0<u_{1}<\Lambda<u_{s}$

Then $\quad d(p)=n \quad, \quad$ and $\quad X(t)=\frac{1}{A_{0}(-1)}$

$Y(t)=-\frac{A_{0}\left(t^{2}\right)-A_{0}(-1)}{A_{0}(-1)\left(1+t^{2}\right)}$,

Which $A_{0}(u)=2 u\left(u-u_{1}^{2}\right) \Lambda\left(u-u_{s}^{2}\right)$

The demonstration is omitted .

\section{Relevant Experiments}

In the following experiments, red curve stands for real curve ,and blue curve stands for interpolation curve . star signal data point and $p(t)$ is interpolation curve $r(t)$ is interpolated curve and $u$ is interval of interpolation . Experiment $1: \mathrm{n}=3 \mathrm{u}=0.0$

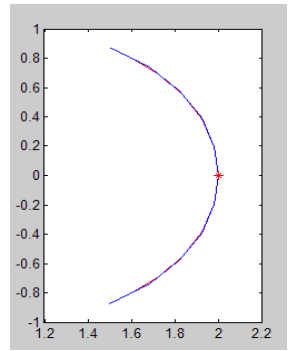

Figure 1. Approximation of the interpolation curves in experiment one

\begin{tabular}{|c|c|c|c|c|c|}
\hline $\mathrm{u}$ & -0.4 & -0.3 & -0.2 & -0.1 & 0 \\
\hline \multirow[t]{2}{*}{$\mathrm{R}(\mathrm{t})$} & 1.7241 & 1.8349 & 1.9231 & 1.9802 & 2.0000 \\
\hline & -0.6897 & -0.5505 & -0.3846 & -0.1980 & 0 \\
\hline \multirow[t]{2}{*}{$\mathrm{P}(\mathrm{t})$} & 1.6800 & 1.8200 & 1.9200 & 1.9800 & 2.0000 \\
\hline & -0.7360 & -0.5730 & -0.3920 & -0.1990 & 0 \\
\hline $\mathrm{u}$ & 0.4 & 0.3 & 0.2 & 0.1 & \\
\hline \multirow[t]{2}{*}{$\mathrm{R}(\mathrm{t})$} & 1.7241 & 1.8349 & 1.9231 & 1.9802 & \\
\hline & 0.6897 & 0.5505 & 0.3846 & 0.1980 & \\
\hline \multirow[t]{2}{*}{$\mathrm{P}(\mathrm{t})$} & 1.6800 & 1.8200 & 1.9200 & 1.9800 & \\
\hline & 0.7360 & 0.5730 & 0.3920 & 0.1990 & \\
\hline
\end{tabular}
$\mathrm{E}=0.0156$

TABLE I RESULT OF THE INTERPOLATION CURVES MATCHING IN FIG.

Experiment $2: \mathrm{n}=3 \mathrm{u}=0.5$ 


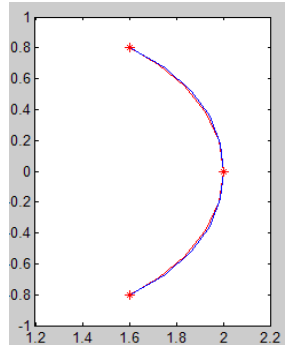

Figure 2. Approximation of the interpolation curves in experiment two

\section{$\mathrm{E}=9.8316 \mathrm{e}-005$}

TABLE II RESULT OF THE INTERPOLATION CURVES MATCHING IN FIG.2

\begin{tabular}{|l|l|l|l|l|l|}
\hline $\mathrm{u}$ & -0.5 & -0.3 & -0.2 & -0.1 & 0 \\
\hline $\mathrm{R}(\mathrm{t})$ & 1.6000 & 1.8349 & 1.9231 & 1.9802 & 2.0000 \\
\cline { 2 - 6 } & -0.8000 & -0.5505 & -0.3846 & -0.1980 & 0 \\
\hline $\mathrm{P}(\mathrm{t})$ & 1.6000 & 1.8613 & 1.9392 & 1.9850 & 2.0000 \\
\cline { 2 - 6 } & -0.8000 & -0.5200 & -0.3542 & -0.1793 & 0 \\
\hline $\mathrm{u}$ & 0.5 & 0.3 & 0.2 & 0.1 & \\
\hline $\mathrm{R}(\mathrm{t})$ & 1.6000 & 1.8349 & 1.9231 & 1.9802 & \\
\cline { 2 - 6 } & 0.8000 & 0.5505 & 0.3846 & 0.1980 & \\
\hline $\mathrm{P}(\mathrm{t})$ & 1.6000 & 1.8613 & 1.9392 & 1.9850 & \\
\cline { 2 - 6 } & 0.8000 & 0.5200 & 0.3542 & 0.1793 & \\
\hline
\end{tabular}

Experiment $3: \mathrm{n}=5 \mathrm{u}=(0.0,0.0)$

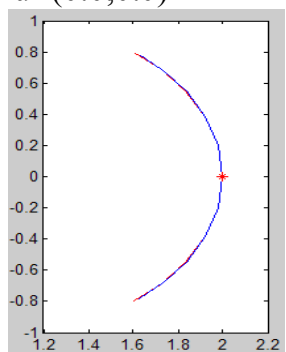

Figure 3. Approximation of the interpolation curves in experiment three $\mathrm{E}=7.0496 \mathrm{e}-005$

TABLE III. RESULT OF THE INTERPOLATION CURVES MATCHING IN FIG.3

\begin{tabular}{|l|l|l|l|l|l|}
\hline $\mathrm{u}$ & -0.5 & -0.3 & -0.2 & -0.1 & 0 \\
\hline $\mathrm{R}(\mathrm{t})$ & 1.6000 & 1.8349 & 1.9231 & 1.9802 & 2.0000 \\
\cline { 2 - 6 } & -0.8000 & -0.5505 & -0.3846 & -0.1980 & 0 \\
\hline $\mathrm{P}(\mathrm{t})$ & 1.6250 & 1.8362 & 1.9232 & 1.9802 & 2.0000 \\
\cline { 2 - 6 } & -0.7813 & -0.5484 & -0.3843 & -0.1980 & 0 \\
\hline $\mathrm{u}$ & 0.5 & 0.3 & 0.2 & 0.1 & \\
\hline $\mathrm{R}(\mathrm{t})$ & 1.6000 & 1.8349 & 1.9231 & 1.9802 & \\
\cline { 2 - 6 } & 0.8000 & 0.5505 & 0.3846 & 0.1980 & \\
\hline \multirow{3}{*}{$\mathrm{P}(\mathrm{t})$} & 1.6250 & 1.8362 & 1.9232 & 1.9802 & \\
\cline { 2 - 6 } & 0.7813 & 0.5484 & 0.3843 & 0.1980 & \\
\hline
\end{tabular}

Experiment $4: \mathrm{n}=5 \mathrm{u}=(0.5,0.5)$

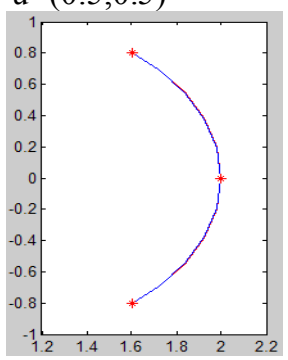

Figure 4. Approximation of the interpolation curves in experiment four $\mathrm{E}=3.2747 \mathrm{e}-005$
TABLEIV. RESULT OF THE INTERPOLATION CURVES MATCHING IN FIG.4

\begin{tabular}{|l|l|l|l|l|l|}
\hline \multicolumn{1}{|c|}{$\mathrm{u}$} & -0.5 & -0.3 & -0.2 & -0.1 & 0 \\
\hline $\mathrm{R}(\mathrm{t})$ & 1.6000 & 1.8349 & 1.9231 & 1.9802 & 2.0000 \\
\cline { 2 - 7 } & -0.8000 & -0.5505 & -0.3846 & -0.1980 & 0 \\
\hline $\mathrm{P}(\mathrm{t})$ & 1.6000 & 1.8376 & 1.9252 & 1.9809 & 2.0000 \\
\cline { 2 - 7 } & -0.8000 & -0.5464 & -0.3794 & -0.1944 & 0 \\
\hline $\mathrm{u}$ & 0.5 & 0.3 & 0.2 & 0.1 & \\
\hline $\mathrm{R}(\mathrm{t})$ & 1.6000 & 1.8349 & 1.9231 & 1.9802 & \\
\cline { 2 - 7 } & 0.8000 & 0.5505 & 0.3846 & 0.1980 & \\
\hline $\mathrm{P}(\mathrm{t})$ & 1.6000 & 1.8376 & 1.9252 & 1.9809 & \\
\cline { 2 - 6 } & 0.8000 & 0.5464 & 0.3794 & 0.1944 & \\
\hline
\end{tabular}

Experiment $5: \mathrm{n}=5 \mathrm{u}=(0.25,0.5)$

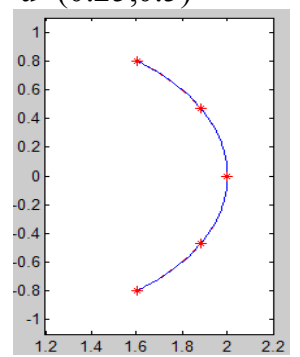

Figure 5. Approximation of the interpolation curves in experiment five

$\mathrm{E}=7.1283 \mathrm{e}-006$

TABLEV. RESULT OF THE INTERPOLATION CURVES MATCHING IN FIG.5

\begin{tabular}{|l|l|l|l|l|l|}
\hline $\mathrm{u}$ & -0.5 & -0.25 & -0.2 & -0.1 & 0 \\
\hline $\mathrm{R}(\mathrm{t})$ & 1.6000 & 1.8824 & 1.9231 & 1.9802 & 2.0000 \\
\cline { 2 - 6 } & -0.8000 & -0.4706 & -0.3846 & -0.1980 & 0 \\
\hline $\mathrm{P}(\mathrm{t})$ & 1.6000 & 1.8824 & 1.9234 & 1.9804 & 2.0000 \\
\cline { 2 - 6 } & -0.8000 & -0.4706 & -0.3840 & -0.1971 & 0 \\
\hline $\mathrm{u}$ & 0.5 & 0.25 & 0.2 & 0.1 & \\
\hline $\mathrm{R}(\mathrm{t})$ & 1.6000 & 1.8824 & 1.9231 & 1.9802 & \\
\cline { 2 - 6 } & 0.8000 & 0.4706 & 0.3846 & 0.1980 & \\
\hline $\mathrm{P}(\mathrm{t})$ & 1.6000 & 1.8824 & 1.9234 & 1.9804 & \\
\cline { 2 - 6 } & 0.8000 & 0.4706 & 0.3840 & 0.1971 & \\
\hline
\end{tabular}

Experiment 6: $\mathrm{n}=9 \mathrm{u}=(0.125,0.25,0.375,0.5)$

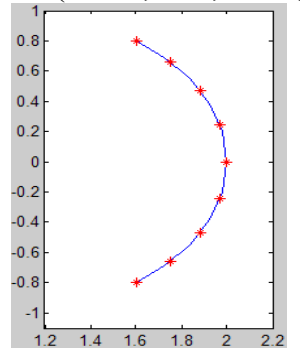

Figure 6. Approximation of the interpolation curves in experiment six

$\mathrm{E}=5.6691 \mathrm{e}-010$

TABLEVI RESULT OF THE INTERPOLATION CURVES MATCHING IN FIG.6

\begin{tabular}{|l|l|l|l|l|l|}
\hline $\mathrm{u}$ & -0.5 & -0.375 & -0.25 & -0.125 & 0 \\
\hline $\mathrm{R}(\mathrm{t})$ & 1.6000 & 1.7534 & 1.8824 & 1.9692 & 2.0000 \\
\cline { 2 - 6 } & -0.8000 & -0.6575 & -0.4706 & -0.2462 & 0 \\
\hline $\mathrm{P}(\mathrm{t})$ & 1.6000 & 1.7534 & 1.8824 & 1.9692 & 2.0000 \\
\cline { 2 - 6 } & -0.8000 & -0.6575 & -0.4706 & -0.2462 & 0 \\
\hline $\mathrm{u}$ & 0.5 & 0.375 & 0.25 & 0.125 & \\
\hline \multirow{3}{*}{$\mathrm{R}(\mathrm{t})$} & 1.6000 & 1.7534 & 1.8824 & 1.9692 & \\
\cline { 2 - 6 } & 0.8000 & 0.6575 & 0.4706 & 0.2462 & \\
\hline \multirow{2}{*}{$\mathrm{P}(\mathrm{t})$} & 1.6000 & 1.7534 & 1.8824 & 1.9692 & \\
\cline { 2 - 6 } & 0.8000 & 0.6575 & 0.4706 & 0.2462 & \\
\hline
\end{tabular}




\section{REFERENCES}

[1] Michael S.Floater. High Order Approximation of Rational Curves by Polynomial Curves[J].Computer Aided Geometric Design,2006,23:621-628.

[2] Dan wei. Several Problems in the Numerical Approximation[D].Dalian University of Technology,2011.

[3] Wang Renhong. Numerical Approximation[M].Higher Education press, 1999.

[4] Ball,A.A.CONSURF Part 1: Introduction of the conic lofting title. ComPuter Aided Design[J],1974,6(4):243-249.

[5] Qi Duan,Gongxue Xu,Aikui Liu,Xuefu Wang,Fuhua (Frank) Cheng. Constrained Interpolation Using Rational Cubic Spline with Linear Denominators[J]. Korean Journal of Computational and Applied Mathematics,1999 (1).

[6] Sederberg.T.W,KakimotoM..Approximating Rational Curves Using Polynomial Curves[C] . In:Farin G,ed.,NURBS for curve and Surface Design.SIAM,PhiladelPhia,1991,144-158.

[7] Wang G.J,Sederberg $T$ W,Chen F L. On the Convergence of Polynomial Approximation of Rational Functions[J]. J of APProx.Theo, 1997,89:267-288

[8] WANG Zhaoqing,LI Shuping,TANG Bingtao. Polynomial Interpolation Approximations of Arbitrary Continuous Functions [J]. Journal of Shandong Jianzhu University,2007(2).

[9] Michael S.Floater.An O(h2n) Hermite Approximation for Conic Sections[J].Computer Aided Geometric Design,1997,14:135-151
[10] Meng Xiangguo, Wang Renhong. Rational Surface Interval Bezier Surface [J]. Journal of Numerical Methods and Computer Applications,2003(4)

[11] Wei Xin. Some Research about Rational Interpolation method[D].Hebei University of Technology,2012.

\section{ACKNOWLEDGEMENTS}

This work was financially supported by the National Natural Science Foundation of China (61163034, 61373067), the Grassland Excellent Talents Project of Inner Mongolia Autonomous Region ( 2013 ), the supported By Program for Young Talents of Science and Technology in Universities of Inner Mongolia Autonomous Region(NJYT-14-A09), the Inner Mongolia Natural Science Foundation(2013MS0911), the 321 Talents Project the two level of Inner Mongolia Autonomous Region(2010), the Inner Mongolia talent development fund(2011), and the Scientific Research Foundation of Inner Mongolia University For Nationalities(NMD1231) 